\title{
Roadmap for Testing and Validation of Electric Vehicle Communication Standards
}

\author{
Richard Pratt ${ }^{1}$, Francis Tuffner, Krishnan Gowri

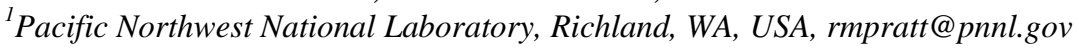

\begin{abstract}
Vehicle to grid communication standards are critical to the charge management and interoperability among plug-in electric vehicles (PEV), charging stations and utility providers. The Society of Automobile Engineers (SAE), International Organization for Standardization (ISO), International Electrotechnical Commission (IEC) and the ZigBee Alliance are developing requirements for communication messages and protocols. While interoperability standards development has been in progress for more than two years, no definitive guidelines are available for the automobile manufacturers, charging station manufacturers or utility backhaul network systems. At present, there is a wide range of proprietary communication options developed and supported in industry. Recent work by the Electric Power Research Institute (EPRI), in collaboration with SAE and automobile manufacturers, has identified performance requirements and developed a test plan based on possible communication pathways using power line communication (PLC). Though the communication pathways and power line communication technology options are identified, much work needs to be done in developing application software and testing of communication modules before these can be deployed in production vehicles. This paper presents a roadmap and results from testing power line communication modules developed to meet the requirements of SAE J2847/1 standard.
\end{abstract}

\section{Introduction}

Communication between PEVs and utilities is required to enable coordinating electric vehicle (EV) charging with power system conditions at the distribution level [1]. Measured data demonstrating the need for coordinating EV charging with power system conditions can be observed from the Idaho National Laboratory (INL) EV project data [2]. The INL EV project provides data on actual charging behavior and Figure 1 shows the U.S. electricity demand impact of 2690 monitored vehicles during the $4^{\text {th }}$ quarter of CY2011. These vehicles are typically configured to begin charging at midnight to take advantage of the period with the best energy price. As additional electric vehicles are added, the change in demand at midnight will be become even more significant.

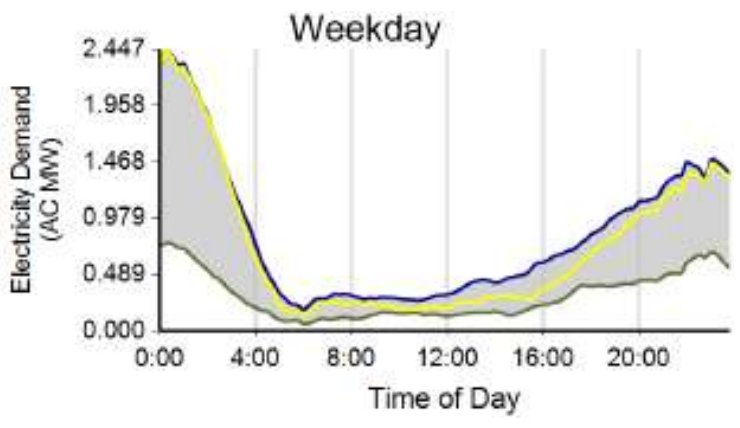

Figure 1: Electricity Demand Impact of Charge Start Time

Information needed to moderate this effect includes vehicle energy needs, vehicle available 
times, electricity price tables, etc. Standardization of this information and communications has been undertaken by SAE, ISO, and IEC. Testing and evaluation of proposed SAE standards has been coordinated and conducted by EPRI and national laboratories. Vehicle and charging station manufacturers, communication system vendors, standards bodies, software developers, and research organizations have contributed to the development of these standards and test plans.

There are three primary standards associated with the communications development roadmap. SAE J1772 specifies the general physical, electrical, functional and performance requirements for conductive charging of PEVs in North America, including the charging connector [3]. SAE J2836/1 documents the Use Cases describing the equipment and interactions to support gridoptimized AC or DC energy transfer for plug-in vehicles. These Use Cases enable Plug-In Vehicles to communicate with the utility so that the customer can take advantage of various incentive programs and charge their PEVs at times and rates to meet their needs [4]. The J2836/1 Use Cases are divided into enrollment, utility programs, connection locations, and charging processes. The J2836/1 Use Case summary is shown in Figure 2.

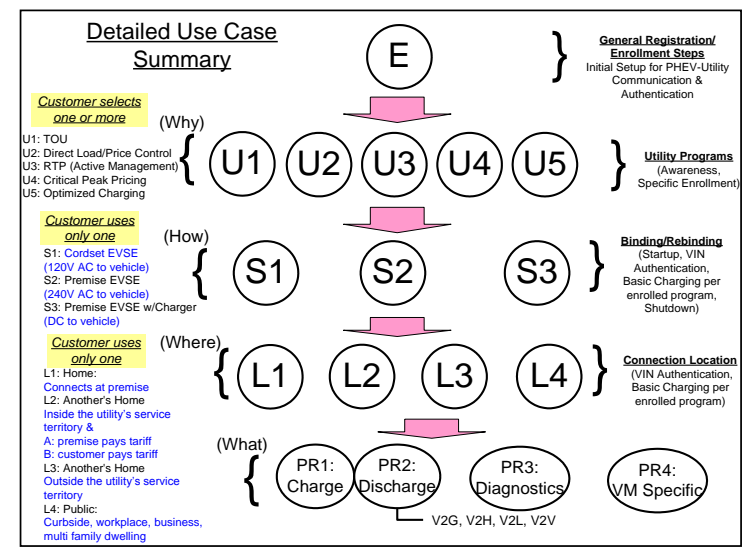

Figure 2: SAE J2836/1 Use Case Summary

The information flow and messages contained in SAE J2847/1 implement the SAE J2836/1 Use Cases. J2847/1's primary purpose is gridoptimized energy transfer for plug-in electric vehicles, and to ensure vehicle operators have sufficient energy for driving while enabling the delivery of that energy to vehicles in ways that minimize stress upon the grid. This can be accomplished, for example, by vehicle owners' voluntary participation in a utility controlledcharging program in return for incentives [5].

There are several mediums and pathways for communications. SAE standards committees focused on using Power Line Carrier (PLC) as the primary medium and using the communication path from the PEV to the electric vehicle supply equipment (EVSE) or charging station. PLC was selected as the primary medium since a direct association from the PEV to the utility can be obtained and is required by some utility programs for special rates or options [5]. Within the EVSE to PEV electrical path, there are two physical layer communication options - the J1772 Control Pilot circuit or the mains (AC or DC power circuit). The Control Pilot circuit is a low voltage circuit used for communicating the maximum charge rate the EVSE can supply to the PEV. The J1772 Connector is shown in Figure 3.

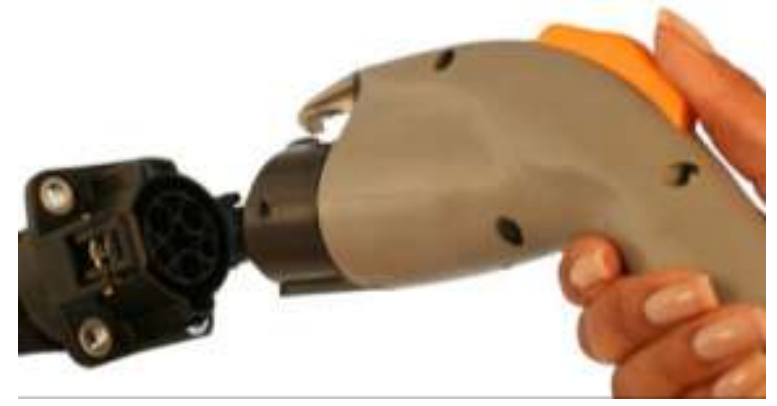

Figure 3: J1772 Connector

SAE and EPRI coordinated the development of J2931/1 test plan [6] to evaluate the performance of communication hardware through a series of tests to vet various communications technologies for automotive application. Communications for two primary purposes were tested: utility/customer communications to support smart charging; and communications to support the use of off-board DC charging equipment. Figure 4 shows the typical communications test configuration used for J2931/1 testing.

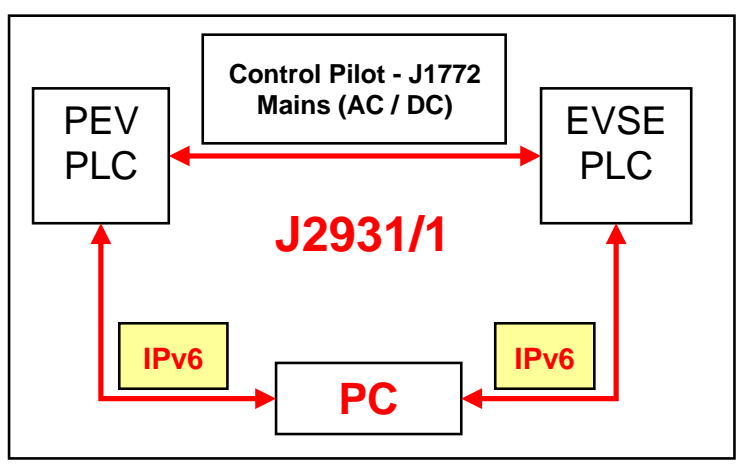

Figure 4: J2931/1 Test Plan Configuration 
Development of the J2931/1 tests started with mapping of requirements from J1772, J2936, and J2847 into two groups - testable and nontestable. Applicable requirements from ISO / IEC documents, commissioned analysis efforts, and initial communication test results were added to the mapping. The broad J2931/1 test categories were control pilot impairment, throughput, latency, crosstalk, co-existence, interference, and shared network. Measurable performance metrics were determined for each category.

Prior to the development of J2931/1 test plan, Pacific Northwest National Laboratory (PNNL) began development of a laboratory test bench and functional testing based on the review of J2936/1 use cases. This paper describes the PNNL effort, test setup and methodology for the technologies tested prior to J2931/1 test plan and also results from testing based on J2931/1 test plan for low-frequency PLC technologies.

\section{Approach}

To investigate communication capabilities and vulnerabilities, PNNL developed a test plan, built testing infrastructure, and conducted testing. All tests were carried out in an identical manner and variations in tests were limited to a single parameter. The following sections will provide details of testing.

\subsection{Test Plan Development}

The communications test plan developed for testing the Echelon PL3170, MAX2990, and MAX2992 was used prior to the J2931/1 test plan being released for use. The data obtained using the PNNL Test Plan was presented to SAE for consideration in the development of the J2931/1 test plan. The PNNL test plan required identification and development of specifications and capabilities necessary to perform the testing. The following testing requirements were chosen.

- Identify SAE J2836 use case(s) or portion of a use case that would be representative of the most critical communication period of the charging process.

- SAE J1772-compliant electrical connections, cables, and control signals will be used for the testing.

- Identify SAE J2847-compliant messaging suitable to test PLC communication products.
- Build a functional test bed for PLC communications testing including battery charger, Level 2 EVSE, and PLC modules.

An analysis of the SAE J2836/1 communications showed that the highest communications requirements occurred when the J1772 connector was plugged into the PEV. This period is shown as Initial Verification and Startup phases in Figure 5. The PEV ID, customer settable preferences (5), Energy Request, and Energy Schedule are communicated during this period. Other information could also be communicated depending on the Use Case preference.

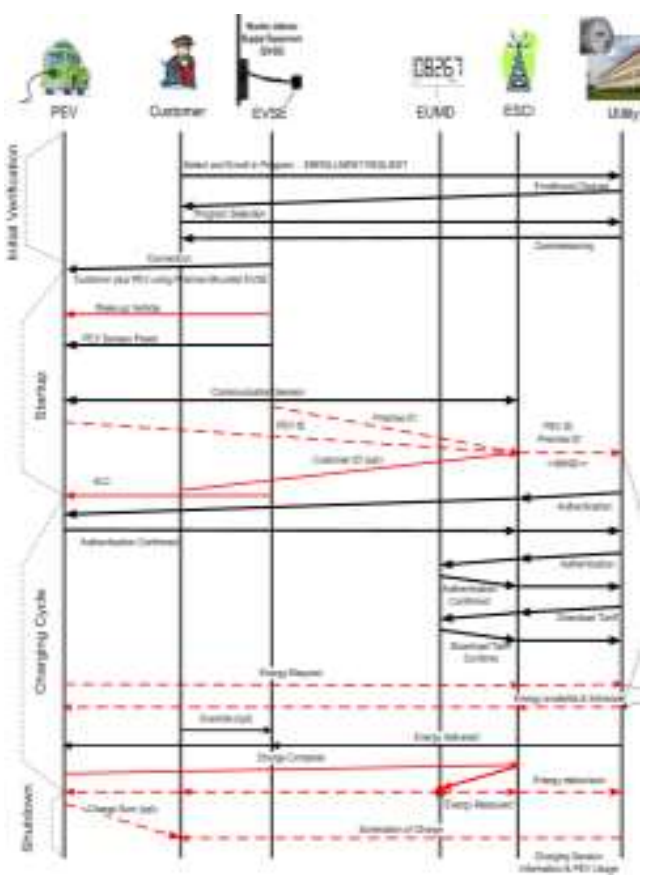

Figure 5: SAE J2836/1 Timing Diagram

The test plan includes test cases, validation criteria, and certification requirements to verify reliability, robustness, repeatability, maximum communication distance, authentication, and security features of V2G communication modules at the application layer level. Other information could be communicated depending on the Use Case preference. Since the longest message (PEV ID) might be up to 20 characters, variable 5character and variable 20-character messages were used to test the communication speed. Five test cases were defined that would best allow reliability, robustness, repeatability, and maximum communication distance to be quantitatively tested. The test cases selected were:

a. Test Case 1: This test case verified PLC transceiver compatibility with the testing infrastructure and made configuration changes 
for each vendor's product. Transmit and receive PLC modules were plugged into the same 120VAC receptacle for this test case.

b. Test Case 2: Evaluate operation on 240 VAC lines with the charger disconnected and operating at $0 \%$ charge rate (idle), $50 \%$ charge rate, $75 \%$ charge rate, $100 \%$ charge rate, and a variable charge rate.

c. Test Case 3: Measure the effect of charging cable length on PLC communications performance.

d. Test Case 4: Evaluate the effect of interfering signals on PLC communications performance.

e. Test Case 5: Tests that highlight the strengths of particular PLC technology.

\subsection{Testing Infrastructure}

The functional test bed was composed of already available laboratory components and required the integration of PLC electronics to a communications test controller, as shown in Figure 6.

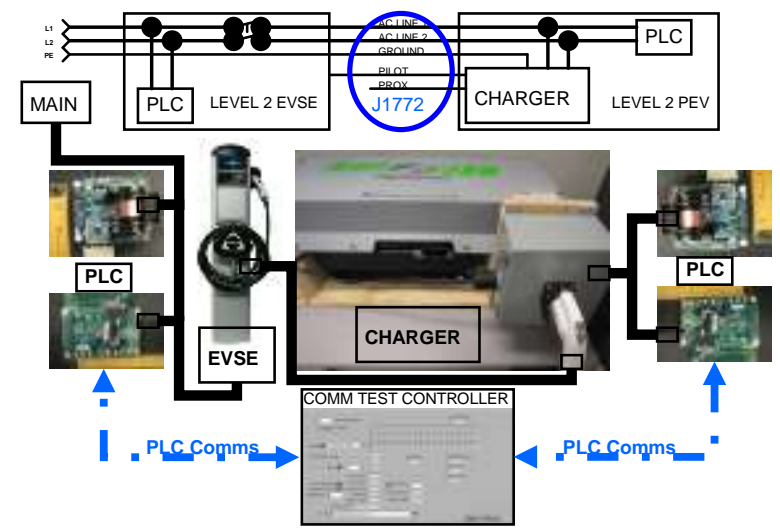

Figure 6: Mains PLC Test Setup

The PLC communication connection to the mains was made inside the Coulomb CT2100 EVSE, and in the adapter box connected to the Hymotion L5 charger. The EVSE contactor was required to be closed for PLC communication on the mains. For PLC communication on the Control Pilot, the contactor need not be closed, but the $1 \mathrm{kHz}$ Control Pilot signal should be present when the contactor is closed.

The PLC products tested used vendor supplied technology demonstration circuit boards. Each board required a different communication interface to enable it to be connected to the PNNL communications controller.

\subsection{Product Configuration}

\subsubsection{Echelon PL3170 Configuration}

The Echelon PL3170 required two levels of configuration. The Echelon solution uses "network variables" located on each PLC module that communicate with each other whenever a network variable's value is changed. These network variables must be programmed on each PLC module. Two network variables were added to each module - a 20-character value representing the vehicle identification number and a 5-character value representing the owner's PIN number. In addition, the Echelon engineers and testing team developed a microcontroller that controlled the PL3170 and allowed it to act as a serial modem. It also operated as a serial communications device with the PNNL communications controller.

\subsubsection{MAX2990 Configuration}

The MAX2990's configuration utility allowed setting the PLC module to be a serial modem with an internal communications delay of 4 milliseconds before forwarding the received packet. The default state is to wait until its $4 \mathrm{kB}$ buffer is full. The MAX2990 uses ROBO mode, a high reliability and lower data rate mode, to improve communication system performance in the presence of high noise conditions.

\subsubsection{MAX2992 Configuration}

The MAX2992's configuration utility allowed setting the PLC module to be a serial modem with an internal communications delay of 6 milliseconds before forwarding the received packet. The default state is to wait until its $4 \mathrm{kB}$ buffer is full. The MAX2992 also uses ROBO mode, a high reliability and lower data rate mode, to improve communication system performance in the presence of high noise conditions.

\subsubsection{Ariane, Tahoe2 and Concerto Test Configurations}

The Ariane Controls, Tahoe2, and Concerto PLC modules were tested using the SAE J2931/1 test plan [6]. The Control Pilot Impairment test measured how this PLC affected the Control Pilot signal both when the PLC was ON and when it was OFF. 


\subsection{Test Descriptions}

The following key definitions are used for testing latency, data rate and bit error rate:

1. Loopback - the time delay between when a message was sent from the transmitting PC and the time in which the message was received by the receiving PC. This eliminates measurement system errors.

2. Latency - the time delay between when a message was sent to the transmitting PLC unit and the time in which the message was received by the receiving PC (Figure 7). The latency time recorded was corrected by subtracting the loopback packet transmission time.

3. J2931/1 Latency - is the round-trip message time, including transmission time and time for the command acknowledgement to be received. The J2931/1 testing used the IPv6 ping command to measure latency.

4. Effective Data Rate - message length (in bits) divided by the latency.

5. Bit Error Rate - the number of bits received in error divided by the total number of bits sent.

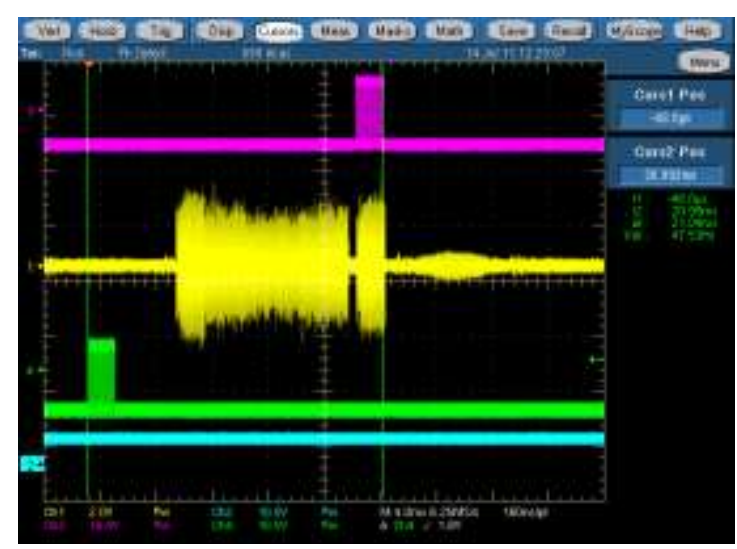

Figure 7: Typical Latency Measurement Technique

\section{Test Results}

\subsection{Echelon PL3170}

Figure 8 shows observed PL3170 PLC signal on the 240VAC mains and the RS232 data transmitted and received from the PL3170 modules.

1. 3.3 million messages were transmitted and received with 58 errors $\left(17 \times 10^{-6} \mathrm{BER}\right)$ and no system lockups. 322 thousand messages were communicated while charging.
2. 193 millisecond latency (195 milliseconds -2 millisecond loopback time).

3. $1.9 \mathrm{Kbps}$ effective data rate

4. Communication error rates and latency were unaffected while using a 30 -foot AC cable in addition to the 17-foot $\mathrm{J} 1772$ cable.

5. Noise injection:

a. The Echelon communication signal was displayed on a spectrum analyzer through an Echelon Power Line Coupling Circuit, Model 78200R. Measurements showed this coupling circuit inserted $0 \mathrm{dBm}$ attenuation from $50 \mathrm{kHz}$ to $500 \mathrm{kHz}$.

b. No errors were observed when an externally generated, FSK signal was added to the power line until the externally generated signal was within $\sim 2 \mathrm{~dB}$ of the Echelon power line signal amplitude. The measured latency increased to 930 milliseconds and the secondary PLC channel signal was visible on the spectrum analyzer.

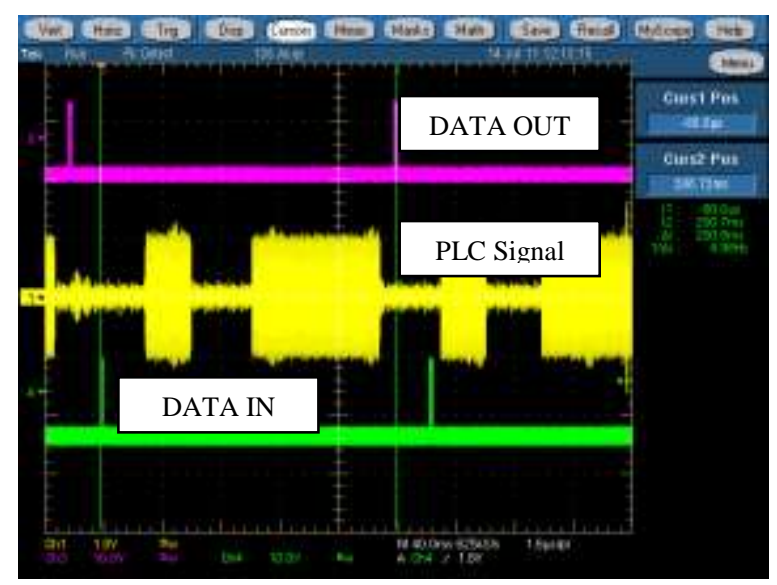

Figure 8: Echelon PL3170 - 40 Byte Message, 200ms Latency

\subsection{MAX2990}

Figure 9 shows observed MAX2990 PLC signal on the 240VAC mains and the RS232 data transmitted and received from the MAX2990 modules. ROBO mode offers a higher reliability, but lower data rate than Normal mode.

- ROBO mode uses a 9-byte packet payload with $\sim 15$ milliseconds between 9-byte payload packets. The MAX2990 uses the RS-232 CTS line to delay payloads more than 9-bytes in ROBO mode.

- Normal mode uses a 140-byte packet payload with $\sim 20$ milliseconds between 140-byte payload 
packets. The MAX2990 uses the RS-232 CTS line to delay payloads more than 140-bytes.

1. 25 million messages were transmitted and received without errors. 2.8 million messages were communicated while charging.

2. Normal Mode Latency - 37 millisecond latency (42 milliseconds -5 millisecond loopback time) for a 20-byte message.

3. ROBO Mode Latency - 50 millisecond latency ( 52 milliseconds -2 millisecond loopback time) for a 20-byte message.

4. The MAX2990 effective data rate is a function of the message length and mode (NORMAL and ROBO). The time out delay is a period of RS-232 inactivity

a. Normal Mode - 20-byte message effective data rate $-4.5 \mathrm{Kbps}$

b. Normal Mode - 251-byte message effective data rate $-33.4 \mathrm{Kbps}$

c. ROBO Mode - 20-byte message effective data rate $-3.6 \mathrm{Kbps}$

d. ROBO Mode - 251-byte message effective data rate $-4+.8 \mathrm{Kbps}$

5. Communication error rates and latency were unaffected while using a 30-foot AC cable in addition to the 17-foot $\mathrm{J} 1772$ cable.

6. ROBO mode was not needed while no other transmitters were on the power line.

7. Noise injection:

a. The MAX2990 communication signal was displayed on a spectrum analyzer through an Echelon Power Line Coupling Circuit, Model 78200R. Measurements showed this coupling circuit inserted no attenuation from $50 \mathrm{kHz}$ to $500 \mathrm{kHz}$.

b. When the similar amplitude signals were added to the power line used in the PL3170 tests, the bit error rate on 882 messages was $136,054 \times 10^{-6}$. Roughly 7 out of 8 messages successfully were received in NORMAL mode. In ROBO mode, the bit error rate returned to 0 .

c. When the function generator output was reduced to 2.4 volts $(-3 \mathrm{~dB})$, the bit error rate returned to zero in NORMAL mode.

d. When the function generator output was increased to 4 volts $(+3 \mathrm{~dB})$, the bit error rate remained at zero in $\mathrm{ROBO}$ mode.

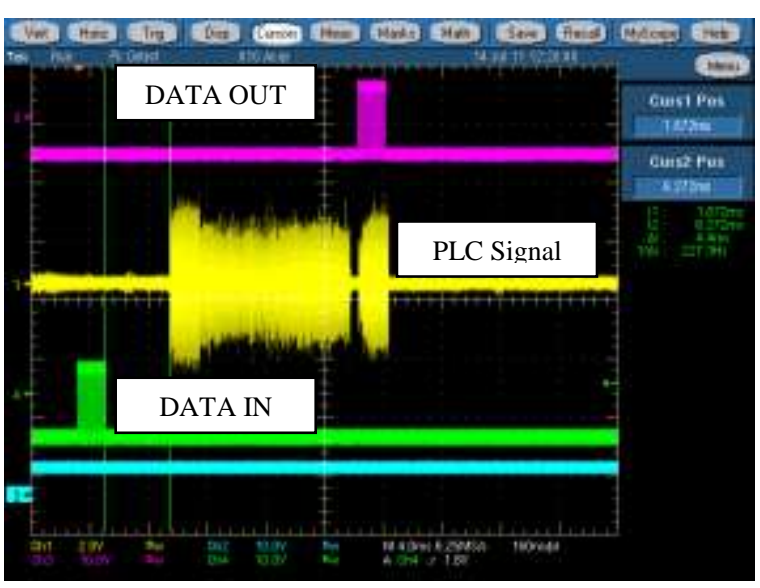

Figure 9: MAX2990 20 Byte Message on 240VAC Mains

\subsection{MAX2992 over Mains}

The MAX2992 was configured to operate on the 240VAC mains. Figure 10 shows the captured signals, using 100-buyte packets. Interference testing was not performed on the MAX2992. The observed data rate in various modulation modes (i.e. DBPSK, DQPSK, or D8PSK) and error rate are below:

1. ROBO Data Rate $=16 \mathrm{Kbps}$

2. DBPSK Data Rate $=21 \mathrm{Kbps}$

3. DQPSK Data Rate $=27.5 \mathrm{Kbps}$

4. D8PSK Data Rate $=28.5 \mathrm{Kbps}$

5. Error Rate $<1 \times 10^{-6}$

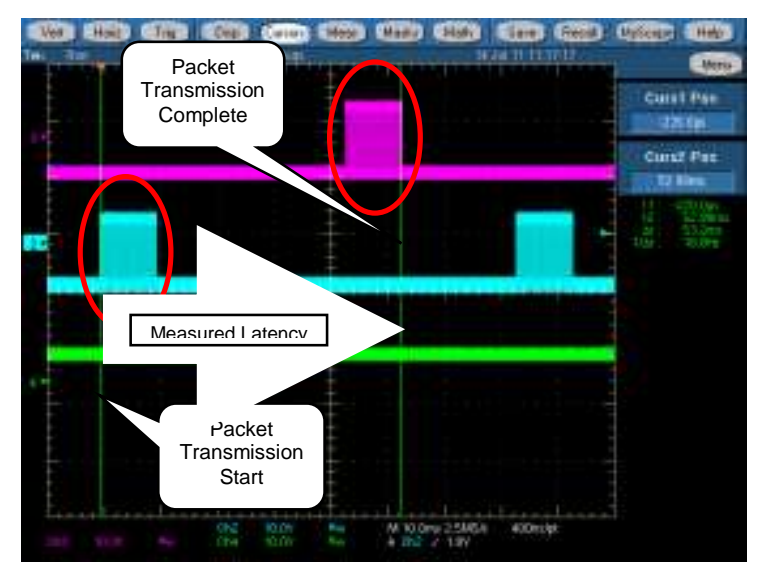

Figure 10: MAX2992 on 240VAC Mains

\subsection{MAX2992 over Control Pilot}

The MAX2992 was configured to operate on the Control Pilot by changing the impedance of the coupling circuit. Captured signals are shown in the Figure 11. The measured PLC signal amplitude was $61 \mathrm{mV}_{\text {rms }}$ and 100-byte packets were used.

1. $\mathrm{ROBO}$ Data Rate $=16 \mathrm{Kbps}$ 
2. DBPSK Data Rate $=25 \mathrm{Kbps}$

3. DQPSK Data Rate $=27.5 \mathrm{Kbps}$

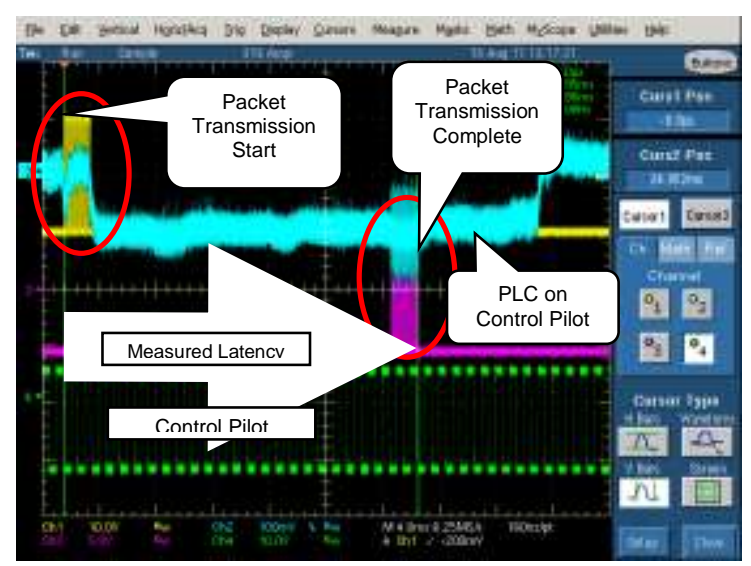

Figure 11: MAX2992 on Control Pilot

\subsection{Ariane Controls AC-CPM1}

The Ariane Controls AC-CPM1 was tested for Control Pilot Impairment, latency, and throughput using SAE J2931/1 procedures. This hardware was supplied on very short notice and functioned well out of the box using RS232, SPI, and Controller Area Network (CAN) interface. The initial communications test results were dramatically improved with a firmware update provided during the testing period. Latency and Throughput were measured using PNNL supplied external IPv4 interface.

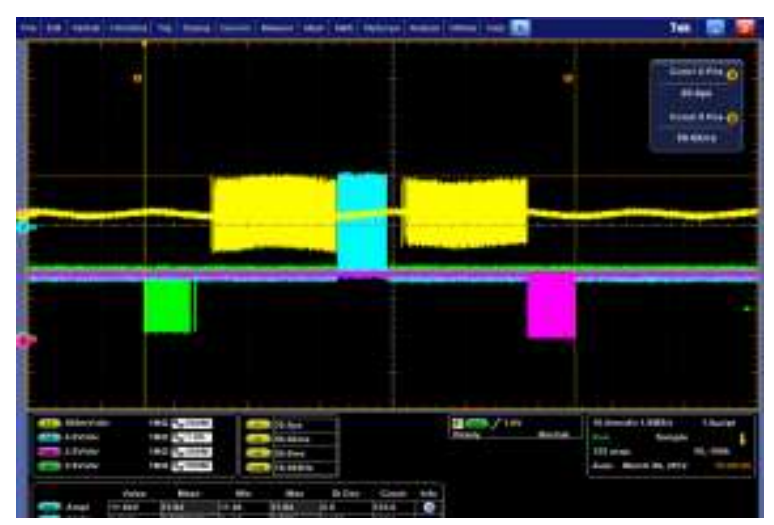

Figure 12: Ariane Controls Signals

Significant test results include:

1. Signal Amplitude $-0.74 \mathrm{~V}_{\mathrm{pp}}$

2. Latency $-47.3 \mathrm{~ms}$. (IPv4 ping)

3. Throughput $-19 \mathrm{Kbps}$ (using IPv4 overhead)

4. Throughput - 39 Kbps (PHY layer)

\subsection{Maxim Tahoe2}

The Maxim Tahoe2 was tested for Control Pilot Impairment using SAE J2931/1 procedures and throughput was measured using the PNNL method (Figure 13). The firmware enabled communications using either RS232 or IPv6 interface. A Controller Area Network (CAN) hardware interface is available for future firmware development.

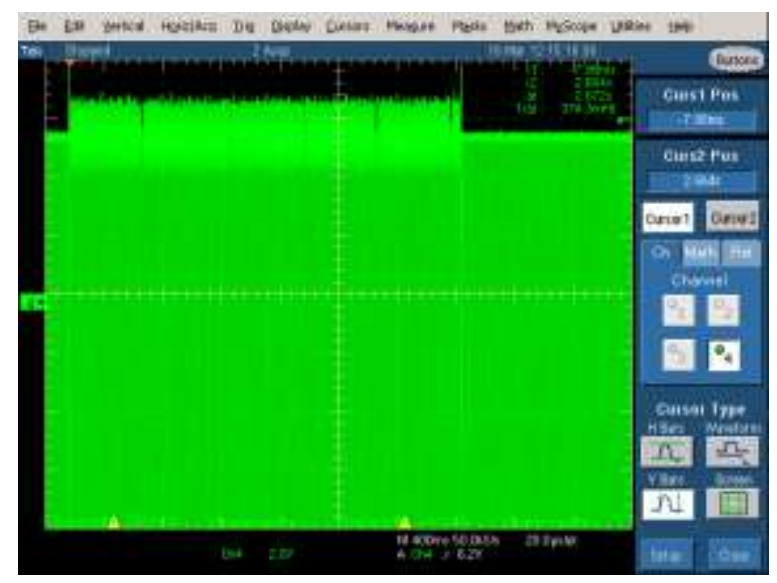

Figure 13: Tahoe2 and Concerto Control Pilot Throughput Measurement

1. Signal Amplitude $-1.24 \mathrm{~V}_{\mathrm{pp}}$

2. Throughput -109 Kbps (PHY layer)

\subsection{TI Concerto}

The Texas Instruments Concerto was tested for Control Pilot Impairment using SAE J2931/1 procedures and throughput was measured using the PNNL method (Figure 13). The firmware provided with the evaluation kit demonstrated communications capability using the vendor supplied GUI. A CAN hardware interface is available for future firmware development.

1. Signal Amplitude $-1.32 \mathrm{Vpp}$

2. Throughput $-105 \mathrm{Kbps}$ (PHY layer)

\subsection{Summary}

Several key results were obtained from completed testing:

1. The high reliability modes used by the PL3170 (secondary channel) and ROBO mode for the MAX2990, MAX2992, and Tahoe2 were effective in maintaining communications when other transmitters or noise existed on the power lines.

2. The messages used for communication throughput measurements are measurably affected by the RS-232 baud rate. 
3. Since the AC-CPM1, MAX2990, MAX2992, Tahoe2, and Concerto use a variable packet length, the message overhead causes the throughput rates to increase as packet size increases.

4. The second generation PLC products (i.e., Tahoe 2 and Concerto) had significantly higher throughput capability than earlier generation products. The addition of hardware interfaces in these second generation products also show preparations are being made to integrate these products into automotive applications.

5. The Ariane Controls throughput testing using IPv4 versus measured physical layer data showed the overhead impact of the IP layer in throughput performance. Increasing the baud rate or implementing an SPI (Serial Peripheral Interface) could reduce this bottleneck.

6. Table 1 shows a summary of all tested products and the performance data. The performance requirements established by J2931/1 for latency and throughput are not met by any of the narrow-band technologies.

Table 1: Product Performance Summary

\begin{tabular}{|c|c|c|c|}
\hline Name & Latency & Error Rate & Throughput \\
\hline \multicolumn{4}{|c|}{ Communication over AC Mains } \\
\hline Echelon PL3170 & $193 \mathrm{~ms}^{(1)}$ & $17 \times 10^{-6}$ & $1.9 \mathrm{Kbps}$ \\
\hline MAX 2990 & $37 m s^{(1)}$ & $<1 \times 10^{-6}$ & $33.4 \mathrm{Kbps}$ \\
\hline MAX 2992 & $17 m s^{(1)}$ & $<1 \times 10^{-6}$ & $28.5 \mathrm{Kbps}$ \\
\hline \multicolumn{4}{|c|}{ Communication over Control Pilot } \\
\hline MAX 2992 & $37 m s^{(1)}$ & $<1 \times 10^{-6}$ & $27.5 \mathrm{Kbps}$ \\
\hline $\begin{array}{c}\text { Ariane AC- } \\
\text { CPM1 }\end{array}$ & $47.4 \mathrm{~ms}^{(2)}$ & ---- & $39 \mathrm{Kbps}$ \\
\hline Maxim Tahoe 2 & ---- & ---- & $109 \mathrm{Kbps}$ \\
\hline TI Concerto & ---- & ---- & $105 \mathrm{Kbps}$ \\
\hline
\end{tabular}

7. PLC communication using the main power lines to the vehicle demonstrated a fairly low data rate for the devices tested. All devices included means for improving communication in a noisy environment, but took a performance hit as noise levels increased. Both the Echelon and Maxim IC devices showed data rates around $30 \mathrm{kbps}$.
8. The J2931/1 tests approached the communication measurement process from the IP layer, where the overhead had a measurable impact. The results highlighted this for all tested devices. With direct physical layer interfaces, data rates were significantly higher (exceeding $100 \mathrm{kbps}$ for newer generation devices). IPv4 testing on the Ariane device showed nearly half the data rate. This test showed the performance reduction related to the overhead of the IP layer.

\section{Conclusions and Further Work}

Several SAE communication standards are emerging to aid in the integration of electric vehicles into the power system. As part of this project, aspects of the $\mathrm{J} 2836 / 1$, J2847, and $\mathrm{J} 1772$ standards were tested using PLC communication devices. The differences in the device interfaces and communication mediums required developing a common test platform to evaluate the communication devices. PNNL met this need by developing a laboratory setup to investigate the different PLC communications devices, as well as providing a platform for testing future electric vehicle communications methods. The application layer communication test architecture, hardware, and measurement methods developed for the narrowband PLC products available can be easily adapted to test other mains or control pilot PLC products, narrowband or broadband PLC products, or ZigBee products.

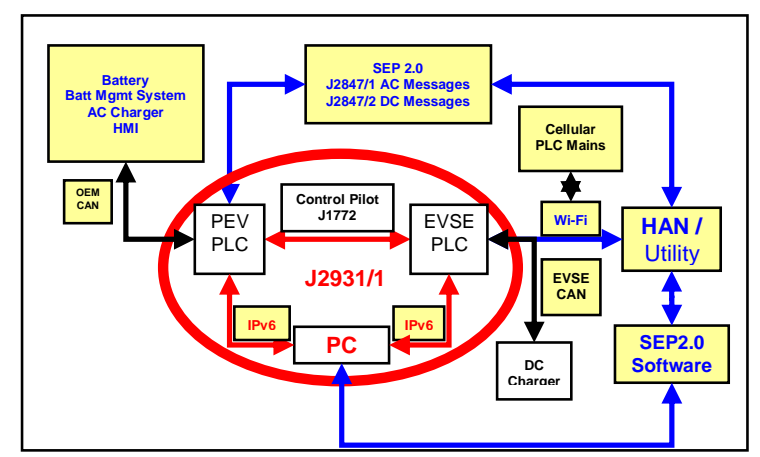

Figure 14: SAE J2931/1 Communication Testing Boundaries

The SAE J2931/1 communication testing described in this paper addresses the physical paths shown circled in Figure 14 . Other important physical paths need to be developed and tested including PEV PLC to vehicle CAN, EVSE PLC to DC Charger, and the EVSE PLC to utility HAN. 
The integration of SEP 2.0 messages onto these physical paths also remains to be developed and tested. The SAE J2931/1 PLC testing and technology selection is nearing completion. Further field testing and ratification of the communication standards are expected by 2014 for wider development and deployment of communication modules.

\section{Acknowledgments}

The authors wish to thank Mr. Vijay Dhingra, Rob Guzikowski and Michael Morrison of Echelon and Jim LeClare, Konrad Scheuer and Kaveh Razazian of Maxim for providing the evaluation kits and assistance during communication module development and testing.

The authors would also like to thank Mr. Matthew Webster and Ed Mullins of Texas Instruments and Jean-Pierre Fournier and Bogdan Baraboi of Ariane Controls for providing the evaluation kits and assistance during communication module development and testing.

The authors would like to acknowledge the support of Mr. Lee Slezak of the U.S. Department of Energy (DOE), Office of Energy Efficiency and Renewable Energy, Vehicle Technologies Program and Mr. Dan Ton of the DOE, Office of Electricity Delivery and Energy Reliability.

\section{References}

[1] Sortomme, E., M. M. Hindi, S. D. J. MacPherson, and S.S. Venkata, "Coordinated Charging of Plug-In Hybrid Electric Vehicles to Minimize Distribution System Losses," in IEEE Transactions on Smart Grid, vol. 2, no. 1, pp. 198-205, Dec 10, 2010.

[2] "EV Project Electric Vehicle Charging Infrastructure Summary Report," Idaho National Laboratory Report, INL/MIS-1121898, February 2012. [Online]. Available: http://avt.inl.gov/pdf/EVProj/EVProjInfrastr uctureQ42011.pdf

[3] SAE J1772 SAE Electric Vehicle and Plug in Hybrid Electric Vehicle Conductive Charge Coupler. (Surface Vehicle Recommended Practice). SAE International, Warrendale, PA.

[4] SAE2836-1 Use Cases for Communication between Plug-in Vehicles and the Utility
Grid. (Surface Vehicle Recommended Practice). SAE International, Warrendale, PA.

[5] SAE J2847-1 Communication between Plugin Vehicles and the Utility Grid. (Surface Vehicle Recommended Practice). SAE International, Warrendale, PA.

[6] J2931/1 PLC Communication Test Plan, September 2011. Electric Power Research Institute, Palo Alto, CA.

\section{Authors}

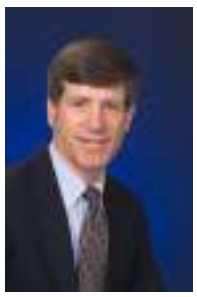

Richard Pratt received a M.S. in Electrical Engineering from the Washington State University in 1988 and a BS in Chemistry from the U.S. Naval Academy. Mr. Pratt is an Engineer at the Pacific Northwest National Laboratory. His research interests include signal processing, PHEV integration, and embedded control systems.

Francis Tuffner received his $\mathrm{Ph} . \mathrm{D}$. in

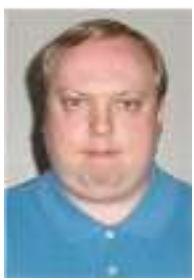
electrical engineering from the University of Wyoming in 2008. He is currently with the Pacific Northwest National Laboratory as a power system engineer. His research interests include signal processing applied to power systems, PHEV integration, embedded control devices, and digital signal processing.

Krishnan Gowri received his Ph.D. in Building Engineering from Concordia

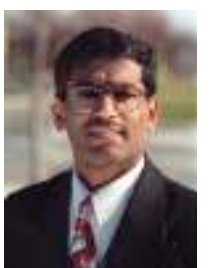
University, Montreal, Que. in 1991. He currently leads the Electric Vehicle Communication Standards and Testing project at PNNL. He participates in SAE Standards committees related to EV communications, and contributes to ASHRAE SPC-201 Facility Smart Grid Information Model standard development. 